\title{
EMPREGO DE SUPORTES DE BAIXO CUSTO PARA IMOBILIZAÇÃO DA LIPASE DE Rhizopus oryzae POR ADSORÇÃO FÍSICA: AVALIAÇÃO DA ESTABILIDADE TÉRMICA
}

\author{
M. P. REINA ${ }^{1}$, L. F. ALVES ${ }^{1}$, A. J. SCHWANKE ${ }^{2}$, A. B. NETO ${ }^{1}$ e A. V. PAULA ${ }^{1}$ \\ ${ }^{1}$ Universidade Estadual Paulista, Faculdade de Ciências Farmacêuticas, Departamento de \\ Bioprocessos e Biotecnlogia \\ ${ }^{2}$ Universidade Federal do Rio Grande do Norte, Centro de Ciências Exatas e da Terra \\ E-mail para contato: marianapradoreina@gmail.com, ariela @fcfar.unesp.br
}

\begin{abstract}
RESUMO - Para o uso de biocatalisadores em larga escala é interessante que eles possam ser reutilizados, sendo a imobilização em suportes sólidos uma importante ferramenta por torná-los insolúveis no meio reacional. Nesse contexto o objetivo deste trabalho foi determinar, dentre diferentes suportes, qual poderia conferir maior estabilidade térmica à lipase de Rhizopus oryzae, imobilizada por adsorção física. Os suportes utilizados foram Caulim e Diatomita (com e sem acidificação). Foram avaliadas as atividades hidrolitica e de esterificação, bem como o rendimento de imobilização. Quanto à atividade hidrolítica, destaca-se o derivado imobilizado em Caulim sem acidificação com ativiadade de 1561,61U/g. Quanto ao rendimento de imobilização, os maiores valores também foram obtidos empregando-se o suporte Caulim sem acidificação (25,68\%). Na atividade de esterificação, o derivado de caulim acidificado resultou em mais elevados valores, $1827,99 \mathrm{U} / \mathrm{g}$. A partir destes resultados, selecionaram-se os derivados imobilizados a serem avaliados quanto à estabilidade térmica nas temperaturas de $45^{\circ} \mathrm{C}, 55^{\circ} \mathrm{C} \mathrm{e}$ $65^{\circ} \mathrm{C}$. O desempenho obtido pelo derivado em Caulim acidificado e em Diatomita acidificada foram muito semelhantes, apresentando o tempo de meia vida em $45^{\circ} \mathrm{C}$ que foi de 18,5 horas e 19,5 horas, respectivamente.
\end{abstract}

\section{INTRODUÇÃO}

As lipases (E.C. 3.1.1.3, triacilglicerol acilhidrolases) são enzimas solúveis em água muito utilizadas biotecnologicamente visando à obtenção de diversos produtos oriundos de reações com lipídeos (Kappor, 2012). São enzimas que catalisam reações de hidrólise e síntese de triglicerídeos, e a versatilidade de reações catalisadas por essas enzimas são o que as tornaram um grupo tão importante biocatalítico no ramo da biotecnologia, com aplicações biotecnológicas e industriais em diversas áreas, tais como a farmacêutica, de detergentes, alimentícia, têxtil, cosmética e de papel (Karl-Erich et al, 2002).

No entanto, para aplicações em larga escala, os biocatalisadores devem ser empregados na forma imobilizada. Isso porque, a imobilização apresenta vantagens como retenção da atividade biológica por mais tempo; facilidade de separação do catalisador e do produto; redução do volume de reação, maior estabilidade ao $\mathrm{pH}$ e a temperatura e possibilidade o 
reaproveitamento da enzima em processos contínuos. As propriedades dos sistemas imobilizados são influenciadas tanto pelas enzimas quanto pelo material do suporte utilizado, sendo que cabe ao suporte a maior contribuição para o desempenho do sistema. Portanto, a escolha de um suporte deve levar em consideração as suas características morfológicas e químicas, bem como a estabilidade química, física e possibilidade de regeneração (Castro et $a l, 2008)$.

Neste trabalho, serão avaliados suportes de baixo custo, que apresentam potencial para imobilização de enzimas. O Caulim é uma argila branca, bastante empregada na indústria de papel devido à sua resistência e alvura e que, justamente por ser natural, tem grande potencial como suporte por atender a demandas ecológicas (Rahman, 2005). Já a Diatomita é uma matéria-prima mineral, originada pela fossilização de algas diatomáceas, composta principalmente por sílica amorfa hidratada $\left(\mathrm{SiO}_{2} \cdot \mathrm{nH}_{2} \mathrm{O}\right)$ ou opalina e impurezas como quartzo, óxidos de ferro, alumínio, sódio potássio, cálcio, magnésio, titânio, matéria orgânica, entre outros. É um material leve e poroso, o que mostra-se como uma importante vantagem quando se trata de imobilização de enzimas (França et al, 2005). Neste contexto, o objetivo deste trabalho foi determinar, dentre diferentes suportes, qual poderia conferir maior estabilidade térmica à lipase de Rhizopus oryzae, imobilizada por adsorção física.

\section{MATERIAIS}

Os experimentos foram efetuados empregando preparação de lipase de Rhizopus oryzae, de grau alimentício gentilmente cedida pela Prozyn (São Paulo). Os suportes utilizados foram Caulim e Diatomita, cedidos pelo Laboratório de Peneiras Moleculares (LABPEMOL) da Universidade Federal do Rio Grande do Norte (UFRN).

\section{METODOLOGIAS}

\subsection{Acidificação}

O processo de acidificação do suporte foi realizado utilizando-se uma solução de ácido nítrico $1 \%$, conforme descrito por Miranda (2004).

\subsection{Imobilização}

O suporte foi imobilizado utilizando meio orgânico (hexano), conforme descrito por Paula (2012).

\subsection{Determinação da Atividade Enzimática de Esterificação}

A atividade de esterificação da enzima foi quantificada pelo consumo de ácido oleico $(12,6 \mathrm{~mL})$ e etanol $(2,4 \mathrm{~mL})$, de acordo com metodologia descrita por Pinto et al (2014).

\subsection{Determinação da Atividade Enzimática de Hidrolítica}

Foi determinada pelo método de retrotitulação, empregando-se emulsão de azeite de oliva, tal como descrito por Paula (2012). 


\subsection{Rendimento de Imobilização}

O rendimento de imobilização foi calculado usando a equação 1:<smiles>[Mg][Mg][Ca][Ca]</smiles>

em que, Uo = unidades de atividade hidrolítica oferecidas para imobilização; U= unidades de atividade hidrolítica total presente no suporte.

\subsection{Estabilidade térmica}

A estabilidade térmica foi determinada pela incubação dos derivados imobilizados em banho termostatizado sem agitação durante $0 \mathrm{~h}, 2 \mathrm{~h}, 4 \mathrm{~h}, 6 \mathrm{~h}$ e $8 \mathrm{~h}$, nas temperaturas de $45^{\circ} \mathrm{C}$, $55^{\circ} \mathrm{C}$ e $65^{\circ} \mathrm{C}$. Após a incubação da enzima nos variados tempos foi efetuada a atividade hidrolítica residual que foi quantificada de acordo com o item 3.5. A atividade relativa (\%) foi calculada de acordo com a equação 2 .

$$
A R(\%)=\frac{U n \times 100}{U_{0}}
$$

em que AR é a atividade relativa no tempo t; Un é a atividade obtida no tempo t e $U_{o}$ é a atividade obtida no tempo 0 horas.

Foi considerada uma cinética de primeira ordem para desativação, sendo a constantes de desativação $\left(K_{d}, \mathrm{~h}^{-1}\right)$ calculadas pela equação 3 e o tempo de meia vida $(t m)$, pela equação 4 :

$$
\ln A=\ln A o-K_{d} * t
$$

em que: $\mathrm{A}_{0}=$ atividade enzimática inicial; $\mathrm{A}=$ atividade residual após tratamento térmico durante um certo período de incubação (t).

$$
t m=\frac{-\ln (0,5)}{K_{d}}
$$

Em que tm é o tempo de meia vida (h) e $\mathrm{K}_{\mathrm{d}}$ é dado $\mathrm{em}^{-1}$.

\section{RESULTADOS E DISCUSSÕES}

\subsection{Atividade Hidrolítica e Rendimento de Imobilização}

Os dados referentes à atividade hidrolítica e ao redimento de imobilização estão apresentados na tabela 1 . 
Tabela 1 - Atividade hidrolítica e rendimento de imobilização dos diversos derivados imobilizados.

\begin{tabular}{|c|c|c|c|c|}
\hline Suporte empregado & Caulim & Caulim Acidificado & Diatomita & Diatomita Acidificada \\
\hline $\begin{array}{c}\text { Atividade Hidrolítica } \\
\text { média } \pm \text { Desvio } \\
\text { padrão (U/g) }\end{array}$ & $1561,51 \pm 26,93$ & $1409,04 \pm 51,07$ & $1368,66 \pm 18,55$ & $1235,67 \pm 9,56$ \\
\hline $\begin{array}{c}\text { Rendimento de } \\
\text { Imobilização (\%) }\end{array}$ & 25,68 & 23,78 & 21,62 & 20,21 \\
\hline
\end{tabular}

Com relação aos valores de atividade hidrolítica, a análise dos dados da Tabela 1 revela que o derivado imobilizado em Caulim forneceu o valor mais elevado de atividade hidrolítica $(1561,51 \pm 26,93 \mathrm{U} / \mathrm{g})$. Isto representa um aumento de $26 \%$ em atividade hidrolítica, se comparado à Diatomita acidificada, com a qual se obteve atividade de 1235,67 $\pm 9,56 \mathrm{U} / \mathrm{g}$.

O processo de acifidicação do suporte foi realizado visando-se avaliar se um tratamento na superfície do suporte poderia favorecer a imobilização da lipase, fornecendo melhores resultados de rendimento de imobilização. No trabalho de Miranda (2004), realizou-se o tratamento do suporte óxido nióbio para abertura de seus poros, antes de empregá-lo na imobilização da lipase. Entretanto, é possível identificar que essa técnica não alterou significantemente a atividade hidrolítica.

\subsection{Atividade de Esterificação}

Após a avaliação da atividade hidrolítica, realizou-se a quantificação da atividade de esterificação dos derivados imobilizados, e os resultados são apresentados na Tabela 2.

Tabela 2 - Atividade de esterificação dos diversos derivados imobilizados.

\begin{tabular}{|c|c|}
\hline Suporte empregado & Atividade Esterificação (U/g) \\
\hline Caulim & 1109,91 \\
\hline Caulim Acidificado & 1827,99 \\
\hline Diatomita & 908,30 \\
\hline Diatomita Acidificada & 1722,66 \\
\hline
\end{tabular}

É possível notar que, no caso da atividade de esterificação, a acidificação dos suportes favoreceu o aumento na atividade do biocatalisador. Assim, para a etapa de estabilidade térmica foram selecionados Caulim e Diatomita acidificados, uma vez que estes forneceram satisfatórias atividades hidrolítica e de esterificação.

\subsection{Estabilidade Térmica}

Os valores de atividade relativa do derivado imobilizado em Caulim e Diatomita acidificados, em função do tempo, para cada temperatura avaliada é apresentado na Figura 1. 
Figura 1 - Perfil de decaimento da atividade do derivado imobilizado em Caulim e Diatomita acidificados no decorrer do tempo sob diferentes temperaturas, em que $\mathrm{A}$ representa o perfil a $45^{\circ} \mathrm{C}, \mathrm{B}$ a $55^{\circ} \mathrm{C}$ e $\mathrm{C}$ e a $65^{\circ} \mathrm{C}$.
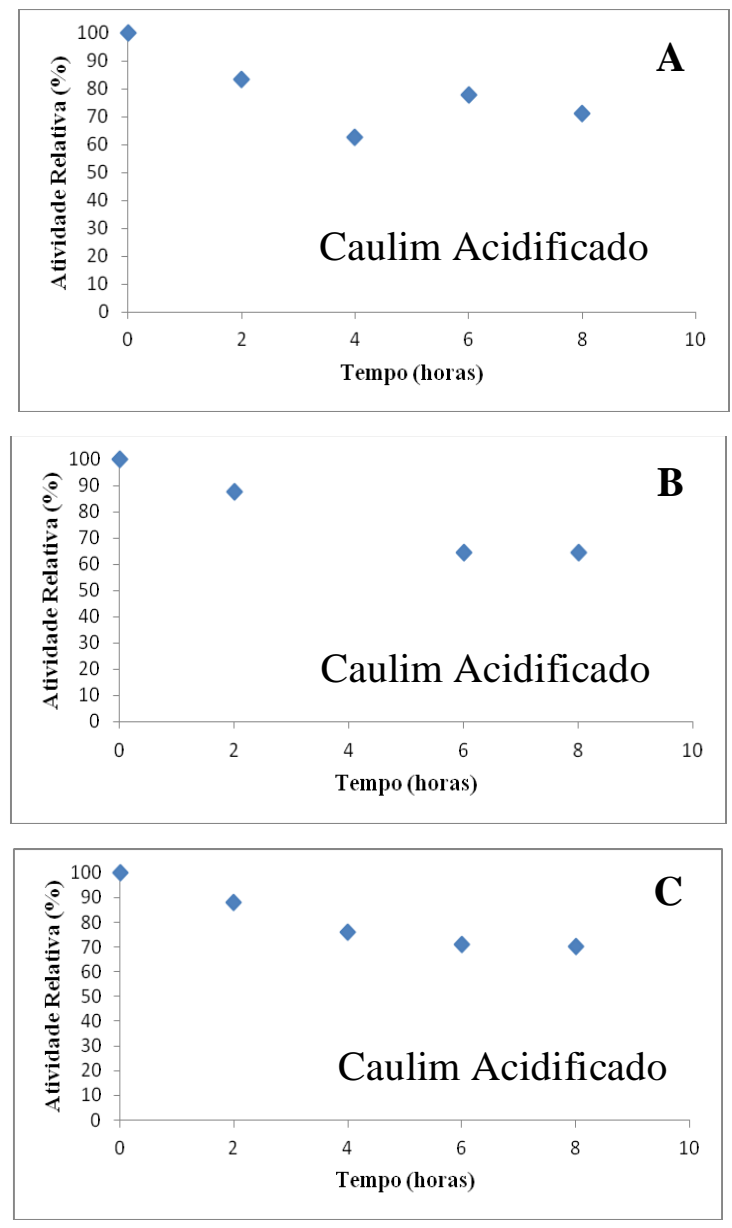
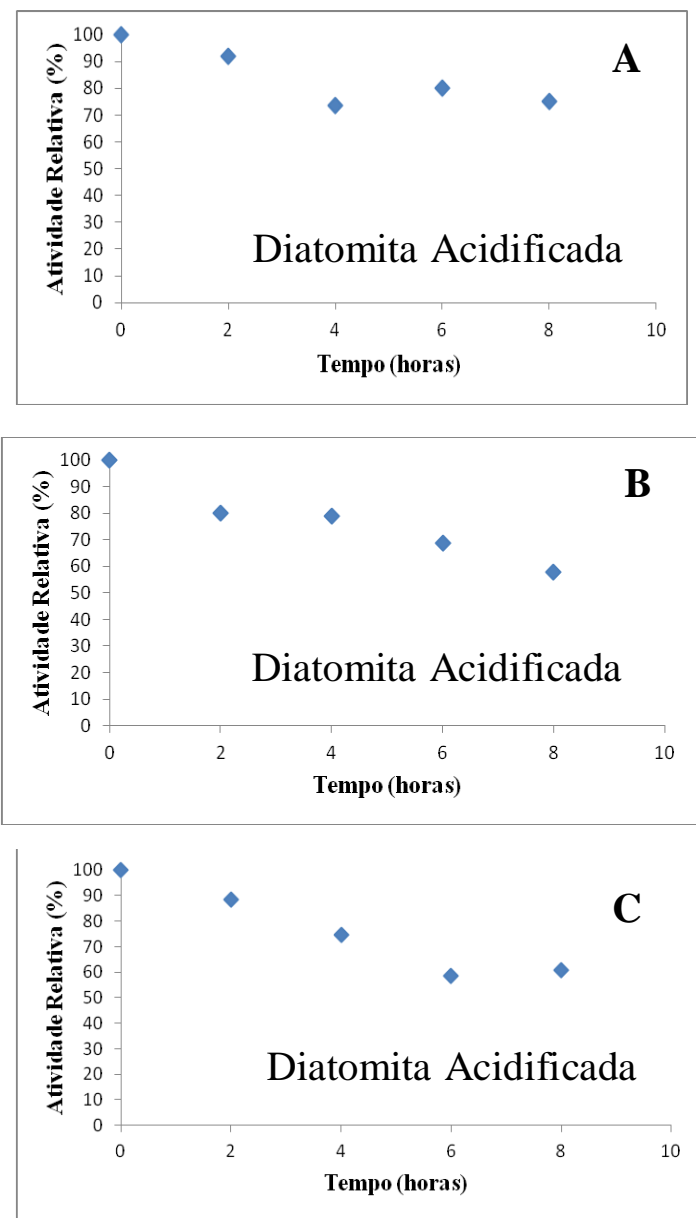

O derivado imobilizado em Caulim apresentou satisfatória estabilidade térmica com valores de atividade hidrolítica que se mantiveram praticamente constantes no decorrer do tempo de incubação. Nas três temperaturas, após as 8 horas ainda havia atividade realtiva de aproximadamente $70 \%$. Quanto à diatomita acidificada, observou-se uma queda na atividade após as 8 horas de incubação e, nesse caso, a temperatura de $45^{\circ} \mathrm{C}$ apresentou uma atividade relativa de $75 \%$, enquanto nas demais temperaturas observou-se uma atividade relativa de aproximadamente $60 \%$.

A partir dos dados de estabilidade térmica, foram calculados os valores de constante de desativação térmica $\left(\mathrm{K}_{\mathrm{d}}\right)$ e tempo de meia vida $\left(\mathrm{t}_{1 / 2}\right)$ da lipase de Rhizopus oryzae em diferentes temperaturas, quando imobilizada nos suportes. Os resultados são apresentados na Tabela 3. Pode-se constatar que os resultados obtidos para os derivados imobilizados nos dois materiais não apresentaram grandes diferenças. $\mathrm{Na}$ temperatura de $45^{\circ} \mathrm{C}$ obtiveram-se os melhores resultados para em ambos os derivados imobilizados e, consequentemente, a maior estabilidade térmica. 
Tabela $3-\mathrm{K}_{\mathrm{d}}$ e tempo de meia vida da lipase de Rhizopus oryzae em diferentes temperaturas, quando imobilizada em diferentes suportes.

\begin{tabular}{|c|c|c|c|c|c|c|}
\hline \multirow{2}{*}{ Suporte } & \multicolumn{2}{|c|}{$\mathbf{4 5}^{\circ} \mathbf{C}$} & \multicolumn{2}{c|}{$\mathbf{5 5}^{\circ} \mathbf{C}$} & \multicolumn{2}{c|}{$\mathbf{6 5}^{\circ} \mathbf{C}$} \\
\cline { 2 - 7 } & ${\mathrm{Kd}\left(\mathrm{h}^{-1}\right)}^{-1} \mathrm{t}_{1 / 2}(\mathrm{~h})$ & $\left.\mathrm{Kd}^{-1} \mathrm{~h}^{-1}\right)$ & $\mathrm{t}_{1 / 2}(\mathrm{~h})$ & $\mathrm{Kd}^{-1}\left(\mathrm{~h}^{-1}\right)$ & $\mathrm{t}_{1 / 2}(\mathrm{~h})$ \\
\hline Caulim Acidificado & 0,037 & 18,48 & 0,073 & 9,39 & 0,059 & 11,79 \\
\hline Diatomita Acidificada & 0,036 & 19,47 & 0,067 & 10,28 & 0,089 & 7,81 \\
\hline
\end{tabular}

\section{CONCLUSÃO}

A imobilização da lipase de Rhizopus oryzae nos suportes apresentados mostrou-se inovadora quando considerado o baixo custo apresentado por esses materiais, permitindo a obtenção de derivados imobilizados com satisfatória estabilidade térmica.

\section{REFERÊNCIAS}

CASTRO, H. F.; ZANIN, G.M.; MORAES, F. F.; SÁ-PEREIRA, P. Imobilização de enzimas e sua estabilização; In: BON, E.P.S.; FERRAR, M. A.; CORVO, M. L; Enzimas em Biotecnologia: produção, aplicações e mercado. Rio de Janeiro: Interciência, p. 123$151,2008$.

FRANÇA, S.C. A., LUZ, A. B., INFORÇATI, P. F.; Diatomita. Rochas e Minerais Industriais. Cetem, p. 300-411, 2005.

KAPOOR, M., GUPTA, M. N. Lipase promiscuity ands it's biochemical applications. Process Biochem. v.47, p. 555-569, 2012

MIRANDA, M. Aprimoramento do processo de imobilização de lipase microbiana em óxido de nióbio para modificação de óleos vegetais. Dissertação de mestrado em Eng. Quím., Universidade de São Paulo, Lorena, 2004.

PAULA, A. V.; Reestruturação da gordura de leite por interesterificação enzimática empregando lipase imobilizada: otimização das condições reacionais e operacionais. Tese de doutorado do programa de pós-graduação de Biotec. Industrial na área de Microbiologia Aplicada, Universidade de São Paulo, Lorena, 2012.

PINTO, M. C. C., FREIRE, D.M.G., PINTO, J. C. Influence of the Morphology of Core-Shell Supports on the Immobilization of Lipase B from Candida antarctica. Molecules, v. 19, p. 12509-12530, 2014.

RAHMAN, M. B. A., TAJUDIN, S. M., HUSSEIN, M. Z., RAHMAN, R. N. Z. A., SALLEH, A. B., BASRI, M. Application of natural kaolin as support for the immobilization of lipase from Candida rugosa as biocatalyst for effective esterification. Appl. Clay Sci., v. 29, p. 111-116, 2005.

\section{AGRADECIMENTOS}

Os autores agradecem à Fundação de Amparo à Pesquisa do Estado de São Paulo (FAPESP-Processo 2016/15748-9) e à Prozyn (São Paulo) pela doação do biocatalisador. 